\title{
Neutrino Oscillations in Matter and Their Applications
}

\author{
Tommy Ohlsson* \\ Institut für Theoretische Physik, Physik Department, Technische Universität München, \\ James-Franck-Straße, DE-85748 Garching bei München, Germany \\ and Division of Mathematical Physics, Theoretical Physics, Department of Physics, \\ Royal Institute of Technology, SE-100 44 Stockholm, Sweden
}

(Dated: February 25, 2001)

In this paper (or talk), an introduction to the evolution operator method for three flavor neutrino oscillations in matter based on the Cayley-Hamilton formalism is presented as well as some its theoretical and experimental applications.

PACS numbers: 14.60.Pq, 14.60.Lm, 13.15.+g, 96.40.Tv

\section{INTRODUCTION}

One can ask oneself questions like: "Why do we study neutrino physics?" and "What is the use of it?". Let us begin and try to motivate such questions with the successes of quantum theory. In the February issue of the journal Scientific American, the following quotation can be found in an article about the 100 years of quantum mysteries written by M. Tegmark and J.A. Wheeler [1]: "Quantum mechanics was instrumental in predicting antimatter, understanding radioactivity (leading to nuclear power), accounting for the behavior of materials such as semiconductors, explaining superconductivity, and describing interactions such as those between light and matter (leading to the invention of the laser) and of radio waves and nuclei (leading to magnetic resonance imaging). Many successes of quantum mechanics involve its extension, quantum field theory, which forms the foundations of elementary particle physics all the way to the present-day experimental frontiers of neutrino oscillations and the search for the Higgs particle and supersymmetry." Having this quotation in mind and especially the underlined phrase, one can just wonder what the outcome of neutrino physics will be!

From quantum mechanics - one of the last century's successes in physics - we know that if neutrinos have masses, then they can oscillate, and vice versa. There are in principle five different types of experiments that are looking for neutrino oscillations occurring in Nature today. These are: solar neutrino oscillation experiments, atmospheric neutrino oscillation experiments, accelerator neutrino oscillation experiments, reactor neutrino oscillation experiments, and cosmic neutrino oscillation experiments. Some of the new experiments are: SNO [2] (solar), BOREXINO [3] (solar), MONOLITH [4, 5](atmospheric), KamLAND [6, 7] (reactor), long baseline (LBL) experiments (such as K2K [8, 9], MINOS [10-12], and CERN-LNGS [13-15]), ICECUBE [16](cosmic) etc. Actually, SNO and K2K are currently in operation.

In general, three flavor neutrino oscillation frameworks should be used when collaborations are analyzing their data, since we today know from LEP data $\left(e^{+} e^{-} \rightarrow Z^{0} \rightarrow \ldots\right)$ that the number of light [43] active neutrino flavors is [17]

$$
N_{\nu}=2.994 \pm 0.012
$$

i.e., $N_{\nu}=3$, and from Big Bang nucleosynthesis $(\mathrm{BBN})\left(\mathrm{BBN}, \mathrm{D} / \mathrm{H}\right.$, and $Y_{p}$ data) we have an upper bound on the number of light $\left(m_{\nu}<1 \mathrm{MeV}\right)$ neutrino species [18]

$$
N_{\nu, \text { light }}<3.2
$$

i.e., there exist (at least) three neutrino flavors and experimental measurements are becoming more and more accurate, which means that we should not loose predictive power when determining oscillation transition probabilities for different channels, mass squared differences, and mixing angles by using oversimplified models for neutrino oscillations, i.e., two flavor neutrino oscillation models.

Using three neutrino flavors, we have three mixing angles (Euler angles) $\theta_{1} \equiv \theta_{23} \equiv \theta_{\text {atm. }}, \theta_{2} \equiv \theta_{13} \equiv \theta_{\mathrm{CHOOZ}}$, and $\theta_{3} \equiv \theta_{12} \equiv \theta_{\odot}$, one $C P$ phase $\delta \equiv \delta_{13} \equiv \delta_{\mathrm{CP}}$, and two mass squared differences $\Delta m^{2} \equiv \Delta m_{21}^{2} \equiv \Delta m_{\odot}^{2}$ and

\footnotetext{
*E-mail addresses: tohlsson@physik.tu-muenchen.de,tommy@theophys.kth.se; Homepages: http://www.physik.tu-muenchen.de/

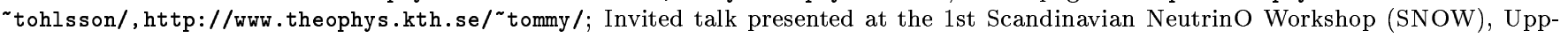
sala, Sweden, February 8-10, 2001. To appear in the Proceedings published jointly by the Royal Swedish Academy of Sciences and the Physical Societies of the five Nordic countries in the international journal Physica Scripta.
} 
$\Delta M^{2} \equiv \Delta m_{32}^{2} \equiv \Delta m_{\text {atm. }}^{2}$, i.e., in total six parameters. If we assume a hierarchical neutrino mass spectrum (also called the normal neutrino mass spectrum), i.e., the neutrino mass eigenstate masses $m_{a}$, where $a=1,2,3$, fulfill the condition

$$
m_{1} \ll m_{2} \ll m_{3},
$$

then $\Delta m_{32}^{2} \simeq \Delta m_{31}^{2}$. In comparison with two neutrino flavors, six parameters are rather many, since for two neutrino flavors there are only two parameters, i.e., one mixing angle $\theta$ and one mass squared difference $\Delta m^{2}$.

In order for the analyses to become realistic, we need also to take into account matter effects. This means that a potential term for the interaction of electron neutrinos with ordinary matter needs to be added to the total Hamiltonian: $V^{\nu_{e}} \simeq \frac{1}{\sqrt{2}} G_{F} \frac{1}{m_{N}} \rho$, where $G_{F} \simeq 1.16639 \cdot 10^{-23} \mathrm{eV}^{-2}$ is the Fermi weak coupling constant, $m_{N} \simeq 939 \mathrm{MeV}$ is the nucleon mass, and $\rho$ is the matter density. (See the talk of H. Snellman for an introduction to the phenomenology of neutrino oscillations [19].)

The outline of this paper is as follows: In Sec. II, we present a short introduction to the evolution operator method for neutrino oscillations in matter based on the Cayley-Hamilton formalism, and in Sec. III, we give the expressions for the neutrino oscillation probability amplitudes in matter as well as the corresponding neutrino oscillation probabilities in matter. Then, in Sec. IV, we discuss the auxiliary matter mixing parameters. Applications to neutrinos traversing the Earth are investigated in Sec. V. Theoretical applications of the evolution operator method such as interference effects between the small and large mass squared differences $\Delta m^{2}$ and $\Delta M^{2}$ and neutrino oscillations in matter of varying density are studied in Secs. VI and VII, respectively. In Sec. VIII, some of the present and future LBL experiments are mentioned. Finally, in Sec. IX, the summary and conclusions are presented.

\section{THE EVOLUTION OPERATOR METHOD}

In order to find the evolution operator for three flavor neutrino evolution (in flavor basis), we need to solve the Schrödinger equation for neutrinos in flavor basis, i.e.,

$$
i \frac{d}{d t} \psi_{f}(t)=\mathscr{H}_{f} \psi_{f}(t)
$$

However, it turns out to be easier to solve the Schrödinger equation for neutrinos in the mass basis and then transform the solution with a unitary transformation to the flavor basis. The Schrödinger equation in mass basis is given by

$$
i \frac{d}{d t} \psi_{m}(t)=\mathscr{H}_{m} \psi_{m}(t)
$$

where

$$
\psi_{m}=\left(\psi_{a}\right)=\left(\begin{array}{c}
\psi_{1} \\
\psi_{2} \\
\psi_{3}
\end{array}\right)
$$

is the vector containing the components of an arbitrary neutrino state $|\nu\rangle=|\nu(t)\rangle=\sum_{a=1}^{3} \psi_{a}(t)\left|\nu_{a}\right\rangle$ and $\mathscr{H}_{m}=$ $H_{m}+U^{-1} V_{f} U$ is the total Hamiltonian (in mass basis). Here $H_{m}=\operatorname{diag}\left(E_{1}, E_{2}, E_{3}\right)$, where $E_{a}=m_{a}^{2} /\left(2 E_{\nu}\right)$, is the free Hamiltonian (in mass basis) and

$U=U\left(\theta_{1}, \theta_{2}, \theta_{3}, \delta\right)=\left(U_{\alpha a}\right)=\left(\begin{array}{ccc}\cos \theta_{2} \cos \theta_{3} & \sin \theta_{3} \cos \theta_{2} & \sin \theta_{2} e^{-i \delta} \\ -\sin \theta_{3} \cos \theta_{1}-\sin \theta_{1} \sin \theta_{2} \cos \theta_{3} e^{i \delta} & \cos \theta_{1} \cos \theta_{3}-\sin \theta_{1} \sin \theta_{2} \sin \theta_{3} e^{i \delta} & \sin \theta_{1} \cos \theta_{2} \\ \sin \theta_{1} \sin \theta_{3}-\sin \theta_{2} \cos \theta_{1} \cos \theta_{3} e^{i \delta} & -\sin \theta_{1} \cos \theta_{3}-\sin \theta_{2} \sin \theta_{3} \cos \theta_{1} e^{i \delta} & \cos \theta_{1} \cos \theta_{2}\end{array}\right)$

is the neutrino mixing matrix, which relates neutrino state components in flavor and mass bases according to $\psi_{f}=$ $U \psi_{m}$ or $\psi_{\alpha}=\sum_{a=1}^{3} U_{\alpha a} \psi_{a}$. The matter potential is

$$
V_{f}=\left(\begin{array}{ccc}
A & 0 & 0 \\
0 & 0 & 0 \\
0 & 0 & 0
\end{array}\right)
$$

where $A=V^{\nu_{e}}$ is a constant. Note that only one element in this matrix is non-zero. Furthermore, note that $H_{m}$ is diagonal in the mass basis, whereas $V_{f}$ is diagonal in flavor basis. The solution to the Schrödinger equation (with $t \simeq L)$ is formally

$$
\psi_{m}(L)=e^{-i \mathscr{H}_{m} L} \psi_{m}(0)
$$


where $L$ is the neutrino (traveling) path length, i.e., the baseline length.

In order to find an analytical expression for this solution, we have to find $e^{-i \mathscr{H}_{m} L}$. This can be achieved with Cayley-Hamilton's theorem from linear algebra.

Cayley-Hamilton's theorem: Assume that $M$ is an $N \times N$ matrix with eigenvalues $\lambda_{a}$, where $a=1,2, \ldots, N$, then the characteristic equation

$$
\begin{gathered}
\chi(\lambda) \equiv \operatorname{det}(M-\lambda I)=\lambda^{N}+c_{N-1} \lambda^{N-1}+\ldots+c_{1} \lambda+c_{0}=0 \\
\Leftrightarrow \\
M^{N}+c_{N-1} M^{N-1}+\ldots+c_{1} M+c_{0} 1_{N}=0 .
\end{gathered}
$$

Here $c_{n}(n=0,1, \ldots, N-1)$ are the so-called principal invariants. Cayley-Hamilton's theorem says that every matrix satisfies its own characteristic equation.

Using Cayley-Hamilton's theorem $\left(M^{p}=c_{N-1}^{(p)} M^{N-1}+\ldots+c_{1}^{(p)} M+c_{0}^{(p)} 1_{N}\right.$ for any $p \geq N$, where $c_{n}^{(p)}(n=$ $0,1, \ldots, N-1)$ are some coefficients), the exponential of the matrix $M$,

$$
e^{M}=\sum_{n=0}^{\infty} \frac{1}{n !} M^{n} \quad \text { (infinite series) }
$$

can be written as

$$
e^{M}=\sum_{n=0}^{N-1} a_{n} M^{n}, \quad \text { (finite series, } N \text { is the dimension of } M \text { ) }
$$

where $a_{n}(n=0,1, \ldots, N-1)$ are some coefficients to be determined.

This means that when $N=N_{\nu}=3$, we have

$$
e^{-i \mathscr{H}_{m} L} \equiv \phi e^{-i T L}=\phi\left(a_{0} 1_{3}-i a_{1} L T-a_{2} L^{2} T^{2}\right),
$$

where $\phi \equiv e^{-i\left(\operatorname{tr} \mathscr{H}_{m}\right) L / 3}, T \equiv \mathscr{H}_{m}-\frac{1}{3}\left(\operatorname{tr} \mathscr{H}_{m}\right) 1_{3}$, and $\operatorname{tr} \mathscr{H}_{m}=E_{1}+E_{2}+E_{3}+A$. Here $T$ is the traceless total Hamiltonian in mass basis. After some (tedious) calculations, we obtain the evolution operator in mass basis as

$$
e^{-i \mathscr{H}_{m} L}=\phi \sum_{a=1}^{3} \frac{e^{-i \lambda_{a} L}}{3 \lambda_{a}^{2}+c_{1}}\left[\left(\lambda_{a}^{2}+c_{1}\right) 1_{3}+\lambda_{a} T+T^{2}\right],
$$

where the eigenvalues $\lambda_{a}(a=1,2,3)$ of the matrix $T$ are the solutions to the characteristic equation $\lambda^{3}+c_{2} \lambda^{2}+$ $c_{1} \lambda+c_{0}=0$. Here $c_{0} \equiv-\operatorname{det} T, c_{1} \equiv \operatorname{det} T \operatorname{tr} T^{-1}=\frac{1}{2}\left[(\operatorname{tr} T)^{2}-\operatorname{tr} T^{2}\right]$, and $c_{2} \equiv-\operatorname{tr} T=0$. Thus, the evolution operator in flavor basis is given by

$$
U_{f}(L)=e^{-i \mathscr{H}_{f} L}=e^{-i U \mathscr{H}_{m} U^{-1} L}=U e^{-i \mathscr{H}_{m} L} U^{-1}=\phi \sum_{a=1}^{3} \frac{e^{-i \lambda_{a} L}}{3 \lambda_{a}^{2}+c_{1}}\left[\left(\lambda_{a}^{2}+c_{1}\right) 1_{3}+\lambda_{a} \tilde{T}+\tilde{T}^{2}\right]
$$

where $\tilde{T} \equiv U T U^{-1}$ is the traceless total Hamiltonian in flavor basis.

The complete derivation of the evolution operator as it has been presented here with the Cayley-Hamilton formalism can be found in Refs. [20, 21].

\section{NEUTRINO OSCILLATION PROBABILITIES}

In order to obtain the neutrino oscillation probabilities, we need to calculate the matrix elements of the evolution operator in flavor basis and take the absolute values of these and then square them. The oscillation probability amplitude from a neutrino flavor $\alpha$ to a neutrino flavor $\beta$ is defined as

$$
A_{\alpha \beta} \equiv\left\langle\beta\left|U_{f}(L)\right| \alpha\right\rangle, \quad \alpha, \beta=e, \mu, \tau .
$$


Thus, the oscillation probabilities for $\nu_{\alpha} \leftrightarrow \nu_{\beta}$ are given by

$$
P_{\alpha \beta} \equiv\left|A_{\alpha \beta}\right|^{2}=A_{\alpha \beta}^{*} A_{\alpha \beta}, \quad \alpha, \beta=e, \mu, \tau .
$$

Inserting the evolution operator in Eq. (9) into Eq. (11) yields the oscillation probabilities (in matter) [22]

$$
P_{\alpha \beta}=\delta_{\alpha \beta}-4 \sum_{\substack{a=1 \\ a<b}}^{3} \sum_{b=1}^{3} \frac{\left(\lambda_{a}^{2}+c_{1}\right) \delta_{\alpha \beta}+\lambda_{a} \tilde{T}_{\beta \alpha}+\left(\tilde{T}^{2}\right)_{\beta \alpha}}{3 \lambda_{a}^{2}+c_{1}} \frac{\left(\lambda_{b}^{2}+c_{1}\right) \delta_{\alpha \beta}+\lambda_{b} \tilde{T}_{\beta \alpha}^{*}+\left(\tilde{T}^{2}\right)_{\beta \alpha}^{*}}{3 \lambda_{b}^{2}+c_{1}} \sin ^{2} \tilde{x}_{a b}
$$

where $\tilde{x}_{a b} \equiv\left(\lambda_{a}-\lambda_{b}\right) L / 2$. When $C P$ nonconservation is neglected, the complex conjugates of the elements of the traceless Hamiltonian in flavor basis can be omitted. Furthermore, the order of the indices does not matter, i.e., $\tilde{T}_{\beta \alpha}=\tilde{T}_{\alpha \beta}$ and $\left(\tilde{T}^{2}\right)_{\beta \alpha}=\left(\tilde{T}^{2}\right)_{\alpha \beta}$.

\section{AUXILIARY MATTER MIXING PARAMETERS}

Introducing the (auxiliary) matter mixing parameters, the oscillation probabilities (in matter) in Eq. (12) can be written as

$$
P_{\alpha \beta}=\delta_{\alpha \beta}-4 \sum_{\substack{a=1 \\ a<b}}^{3} \sum_{\substack{b=1 \\ 3}}^{3}\left(U_{\alpha a}^{M}\right)^{*} U_{\beta a}^{M} U_{\alpha b}^{M}\left(U_{\beta b}^{M}\right)^{*} \sin ^{2} \tilde{x}_{a b},
$$

i.e., we have just added the superscript $M$ to the ordinary formulas for the oscillation probabilities (in vacuum). The oscillation probabilities in Eq. (13) are equivalent ways of writing the oscillation probabilities in Eq. (12).

The auxiliary matter mixing parameters are [22]:

$$
\begin{aligned}
\theta_{1}^{M} & =\arctan \frac{\lambda_{3} \tilde{T}_{\mu e}+\left(\tilde{T}^{2}\right)_{\mu e}}{\lambda_{3} \tilde{T}_{\tau e}+\left(\tilde{T}^{2}\right)_{\tau e}} \\
\theta_{2}^{M} & =\arcsin \sqrt{\frac{\lambda_{3}^{2}+c_{1}+\lambda_{3} \tilde{T}_{e e}+\left(\tilde{T}^{2}\right)_{e e}}{3 \lambda_{3}^{2}+c_{1}}}, \\
\theta_{3}^{M} & =\arctan \sqrt{\frac{\lambda_{2}^{2}+c_{1}+\lambda_{2} \tilde{T}_{e e}+\left(\tilde{T}^{2}\right)_{e e}}{\lambda_{1}^{2}+c_{1}+\lambda_{1} \tilde{T}_{e e}+\left(\tilde{T}^{2}\right)_{e e}} \frac{3 \lambda_{1}^{2}+c_{1}}{3 \lambda_{2}^{2}+c_{1}}}, \\
\delta^{M} & =\frac{i}{2} \ln \frac{\lambda_{3} \tilde{T}_{e \alpha}+\left(\tilde{T}^{2}\right)_{e \alpha}}{\lambda_{3} \tilde{T}_{\alpha e}+\left(\tilde{T}^{2}\right)_{\alpha e}}, \quad \alpha=\mu, \tau .
\end{aligned}
$$

See Fig. 1 for an example of how the behaviors of the auxiliary matter mixing parameters could look like. Note that in this example $\sin ^{2} \theta_{1}^{M}$ becomes larger than 1 for large values of the matter density parameter $A$, which means that $\theta_{1}^{M}$ is complex.

The same physics can thus be described by the two equivalent sets of parameters (representations): $1 . \Delta m^{2}, \Delta M^{2}$, $\theta_{1}, \theta_{2}, \theta_{3}, \delta$, and $A$ or $2 . \widetilde{\Delta m^{2}}, \widetilde{\Delta M^{2}}, \theta_{1}^{M}, \theta_{2}^{M}, \theta_{3}^{M}$, and $\delta^{M}$. However, the definitions of the auxiliary matter mixing parameters given in Eqs. (14) - (17) are not unique. Illuminating approximative formulas of the auxiliary matter mixing parameters for $A / \Delta M^{2} \ll 1$ and $A / \Delta M^{2} \gg 1$ have been derived by M. Freund [24].

\section{APPLICATIONS TO NEUTRINOS TRAVERSING THE EARTH}

The derivation of the evolution operator method in Sec. II has been made under the assumption that we have a constant matter density parameter $A$. However, in reality, most matter density profiles are not constants, but functions that vary with the neutrino path length $L$. For example, there are the matter density profiles of the Earth (step function) and the Sun (exponentially decreasing).

When the neutrinos propagate through a series of $n$ different matter density layers with constant matter density parameters $A_{1}, \ldots, A_{n}$ and thicknesses $L_{1}, \ldots, L_{n}$, respectively, then the total evolution operator is given by [21]

$$
U_{f}(L)=\prod_{k=1}^{n} U_{f}\left(L_{k}, A_{k}\right)=U_{f}\left(L_{n}, A_{n}\right) U_{f}\left(L_{n-1}, A_{n-1}\right) \ldots U_{f}\left(L_{2}, A_{2}\right) U_{f}\left(L_{1}, A_{1}\right)
$$




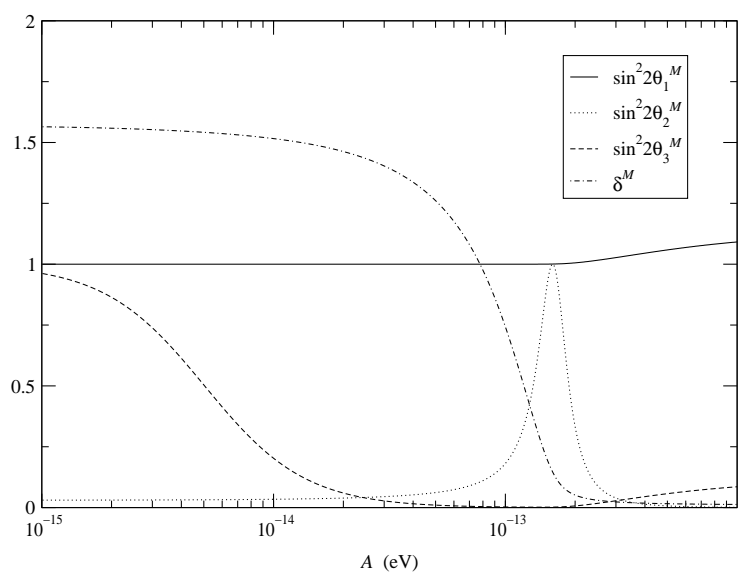

FIG. 1: The auxiliary matter mixing parameters as functions of the matter density parameter $A$, using $\theta_{1}=\theta_{3}=45^{\circ}$ (bimaximal mixing [23]), $\theta_{2}=5^{\circ}, \delta=\pi / 2, \Delta m^{2}=10^{-4} \mathrm{eV}^{2}, \Delta M^{2}=3.2 \cdot 10^{-3} \mathrm{eV}^{2}$, and $E_{\nu}=10 \mathrm{GeV}$.

where

$$
L \equiv \sum_{k=1}^{n} L_{k}
$$

is the total neutrino path length and $U_{f}\left(L_{k}, A_{k}\right)=e^{-i \mathscr{H}_{f}\left(A_{k}\right) L_{k}}$ is the evolution operator in the $k$ th layer. Note that the different $U_{f}\left(L_{k}, A_{k}\right)$ 's do not in general commute with each other, which means that the ordering of them is very important.

Let us take the Earth matter density profile as an example. In Fig. 2, we show the (realistic) Earth matter density profile [25] as well as a mantle-core-mantle step function approximation of this profile. It has been found that the mantle-core-mantle step function model is a very good approximation to the (realistic) Earth matter density profile [26]. Thus, since the mantle-core-mantle step function model consists of three matter density layers (mantle, core, and mantle), we have the evolution operator (for neutrinos crossing the center of the Earth)

$$
U_{f}\left(L_{\oplus}\right)=U_{f}\left(L_{\text {mantle }}, A_{\text {mantle }}\right) U_{f}\left(L_{\text {core }}, A_{\text {core }}\right) U_{f}\left(L_{\text {mantle }}, A_{\text {mantle }}\right),
$$

where $L_{\oplus} \equiv 2 L_{\text {mantle }}+L_{\text {core }}$. We will use the following values of the matter density parameters and the corresponding thicknesses:

$$
\begin{aligned}
& A_{\text {mantle }} \simeq 1.7 \cdot 10^{-13} \mathrm{eV}\left(\rho_{\text {mantle }}=4.5 \mathrm{~g} / \mathrm{cm}^{3}\right), \quad L_{\text {mantle }}=2885 \mathrm{~km}, \\
& A_{\text {core }} \simeq 2.1 \cdot 10^{-13} \mathrm{eV}\left(\rho_{\text {core }}=11.5 \mathrm{~g} / \mathrm{cm}^{3}\right), \quad L_{\text {core }}=6972 \mathrm{~km},
\end{aligned}
$$

even though Fig. 2 shows a mantle-core-mantle step function approximation with $\rho_{\text {mantle }}=5 \mathrm{~g} / \mathrm{cm}^{3}$ and $\rho_{\text {core }}=$ $12 \mathrm{~g} / \mathrm{cm}^{3}$.

Neutrinos traversing the Earth towards a detector that is close to the surface of the Earth propagate in general through the mantle $\left(A_{\text {mantle }}, L_{\text {mantle }}\right)$, the core $\left(A_{\text {core }}, L_{\text {core }}\right)$, and then the mantle again $\left(A_{\text {mantle }}, L_{\text {mantle }}\right)$, where the distances $L_{\text {mantle }}$ and $L_{\text {core }}$ are functions of the nadir angle $h$ (or the zenith angle $\vartheta_{z}$ ). Note that the zenith and nadir angles $\vartheta_{z}$ and $h$ obey the following identity: $\vartheta_{z}+h \equiv \pi=180^{\circ}$. As $h\left(\vartheta_{z}\right)$ varies from $0\left(180^{\circ}\right)$ to $90^{\circ}\left(90^{\circ}\right)$, the cord $L_{\oplus}=L_{\oplus}(h)$ of the neutrino propagation through the Earth becomes shorter and shorter (see Fig. 3). At an angle larger than $h_{0} \equiv \arcsin \frac{r_{\oplus}}{R_{\oplus}} \simeq 33.17^{\circ}$, where $R_{\oplus} \simeq 6371 \mathrm{~km}$ is the radius of the Earth and $r_{\oplus} \simeq 3486 \mathrm{~km}$ is the radius of the core of the Earth, the distance $L_{\text {core }}=0$, and the neutrinos no longer propagate through the core. The 


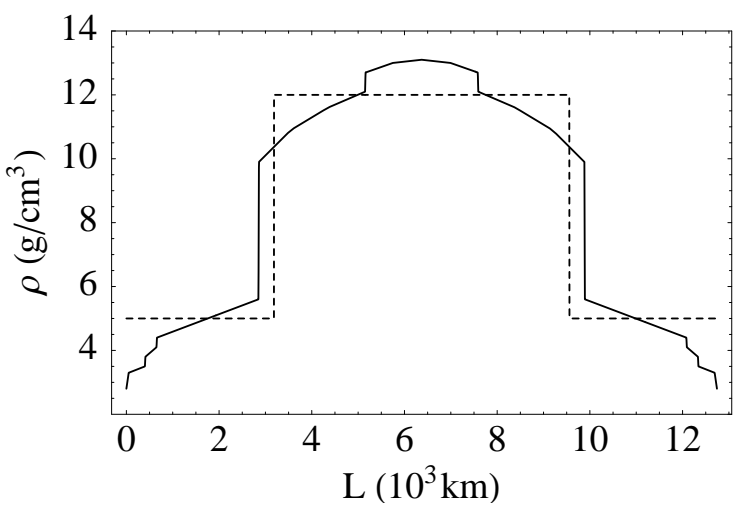

FIG. 2: The Earth matter density profile. The solid curve represents the (realistic) Earth matter density profile [25], whereas the dashed curve represents the mantle-core-mantle step function approximation of the Earth matter density profile. This figure has been taken from Ref. [26].
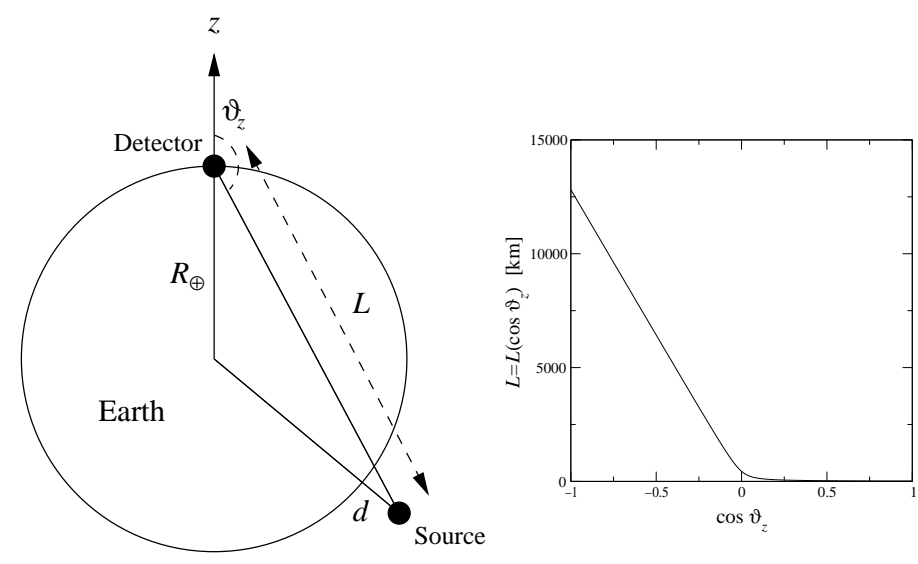

FIG. 3: The left figure shows a schematic picture of the neutrino path length $L$ for atmospheric neutrinos from the production point (source) to the detection point (detector) and the right figure shows a plot of the neutrino path length $L$ as a function of $\cos \vartheta_{z}$, where $\vartheta_{z}$ is the zenith angle. If $\vartheta_{z}=0$, then we have down-going neutrinos and $L=d$, and if $\vartheta_{z}=\pi$, then we have up-going neutrinos and $L=2 R_{\oplus}+d$.

neutrino path length $L_{\oplus}$ as a function of the nadir angle is given by

$$
L_{\oplus} \equiv L_{\oplus}(h)=\left\{\begin{array}{ll}
\underbrace{2 R_{\oplus}\left(\cos h-\sqrt{\left(\frac{r_{\oplus}}{R_{\oplus}}\right)^{2}-\sin ^{2} h}\right)}_{L_{\text {mantle }}(h)}+\underbrace{2 \underbrace{R_{\oplus} \cos h}_{\text {mantle }},}_{L_{\text {core }}(h)}, & h_{0} \leq h \leq 90^{\circ}
\end{array},\right.
$$

which should not be confused with the neutrino path length for atmospheric neutrinos $L_{\text {atm. }}$, i.e.,

$$
L_{\text {atm. }} \equiv L \equiv L\left(\cos \vartheta_{z}\right)=\sqrt{R_{\oplus}^{2} \cos ^{2} \vartheta_{z}+2 R_{\oplus} d+d^{2}}-R_{\oplus} \cos \vartheta_{z},
$$

where $d(10 \mathrm{~km}-20 \mathrm{~km})$ is the altitude of the production point of the atmospheric neutrinos (see Fig. 3). However, when $d$ is small, i.e., $d \ll R_{\oplus}$, it holds that $L_{\oplus} \simeq L_{\text {atm. }}$.

In Fig. 4, as an example of the total evolution operator method, we have plotted the survival oscillation probability $P_{e e}$ as function of the nadir angle $h$ and the neutrino energy $E_{\nu}$, i.e., $P_{e e}=P_{e e}\left(h, E_{\nu}\right)$, for the mantle-core-mantle step function approximation model of the Earth. At $h=h_{0}$, where $h_{0} \simeq 33.17^{\circ}$, the neutrinos no longer traverse the 


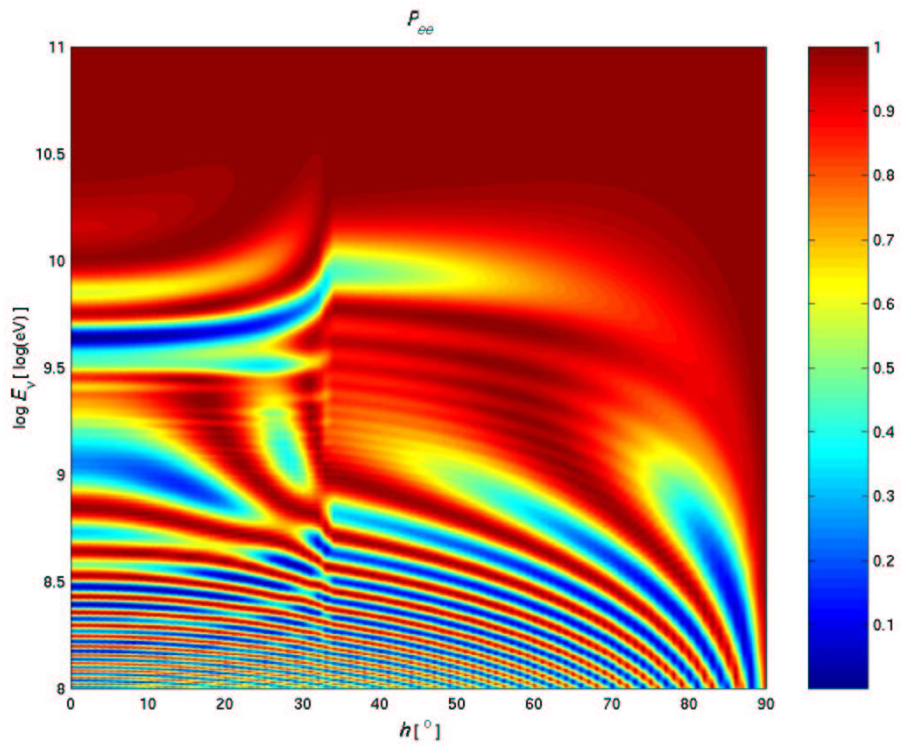

FIG. 4: The (survival) oscillation probability $P_{e e}$ as a function of the nadir angle $h$ and the neutrino energy $E_{\nu}$ for the parameter values $\theta_{1}=\theta_{3}=45^{\circ}$ (bimaximal mixing [23]), $\theta_{3}=5^{\circ}, \delta=0, \Delta M^{2}=3.2 \cdot 10^{-3} \mathrm{eV}^{2}$, and $\Delta m^{2}=\Delta M^{2} / 10$. Dark red corresponds to $P_{e e}=1$ and dark blue corresponds to $P_{e e}=0$.

core of the Earth. In order for the neutrinos also to traverse the core of the Earth, the baseline needs to be at least $L \simeq 10670 \mathrm{~km}$.

Experiments that are well-suited for studying neutrino oscillations in the Earth are: atmospheric neutrino oscillation experiments, LBL neutrino oscillation experiments, and future neutrino factories (or neutrino factory complexes).

Thus, even an LBL experiment from Fermilab to Gran Sasso with a baseline length of approximately $7332 \mathrm{~km}$ would not be enough in order for the neutrino beam also to enter the core.

\section{INTERFERENCE EFFECTS}

In this section, we will discuss interference effects of the two mass squared differences $\Delta m^{2}$ and $\Delta M^{2}$ [44]. These effects are not present in two flavor neutrino oscillation models for obvious reasons. Furthermore, these interference effects have nothing to do with the Mikheyev-Smirnov-Wolfenstein (MSW) effect [27-29], but are solely due to the presence of two mass squared differences and varying matter densities.

The results of our calculations are given in Figs. 5 and 6 [30]. (We have used the mantle-core-mantle step function approximation of the Earth matter density profile.) Figure 5 shows contour plots of $P_{\mu e}$ as a function of $h$ and $\Delta M^{2} / E_{\nu}$. This figure contain 10 subplots for varying small mass squared difference $\Delta m^{2}$. The values of $\Delta m^{2}$ are between 0 and $\Delta M^{2}$ (see the figure caption for the exact values) [45]. In this figure, as $\Delta m^{2}$ increases, we observe that the oscillation pattern becomes more and more complicated. The red (blue) areas correspond to parameter regions of large (small) transition probability $P_{\mu e}$ for the assumed parameter values. When $\Delta m^{2}$ increases its value, oscillatory areas are starting to move down from large values of $\Delta M^{2} / E_{\nu}$. In the same time, the simply connected red area starts to break up into smaller pieces. For some specific values of the nadir angle $h$ and the neutrino energy $E_{\nu}$ considerable drops in the transition probability $P_{\mu e}$ are observed. Furthermore, the maximal transition probability $P_{\mu e}^{\max }$ increases its value. In the limit when $\Delta m^{2}=\Delta M^{2}$ (subplot 10), the distinct red area in subplot 1 is completely dissolved and only an oscillatory pattern is left.

In Fig. 6, the maximal appearance oscillation transition probability $P_{\mu e}^{\max }$ is plotted as a function of the small mass squared difference $\Delta m^{2}$ for some different nadir angles (see the figure caption for details). We observe that the qualitative pattern of the curves do not change very much for different nadir angles. For $\Delta m^{2} \sim 10^{-3} \mathrm{eV}^{2} P_{\mu e}^{\mathrm{max}} \mathrm{can}$ become as large as 0.85 , which means an enhancement of up to as much as $70 \%$. (From studies of solar neutrino data it has been found that the large mixing angle (LMA) solution could be as large as $\Delta m^{2} \simeq 10^{-3} \mathrm{eV}^{2}[31,32]$.) For values of $\Delta m^{2}$ close to $\Delta M^{2}$ and above, the results are only of pure theoretical interest. 

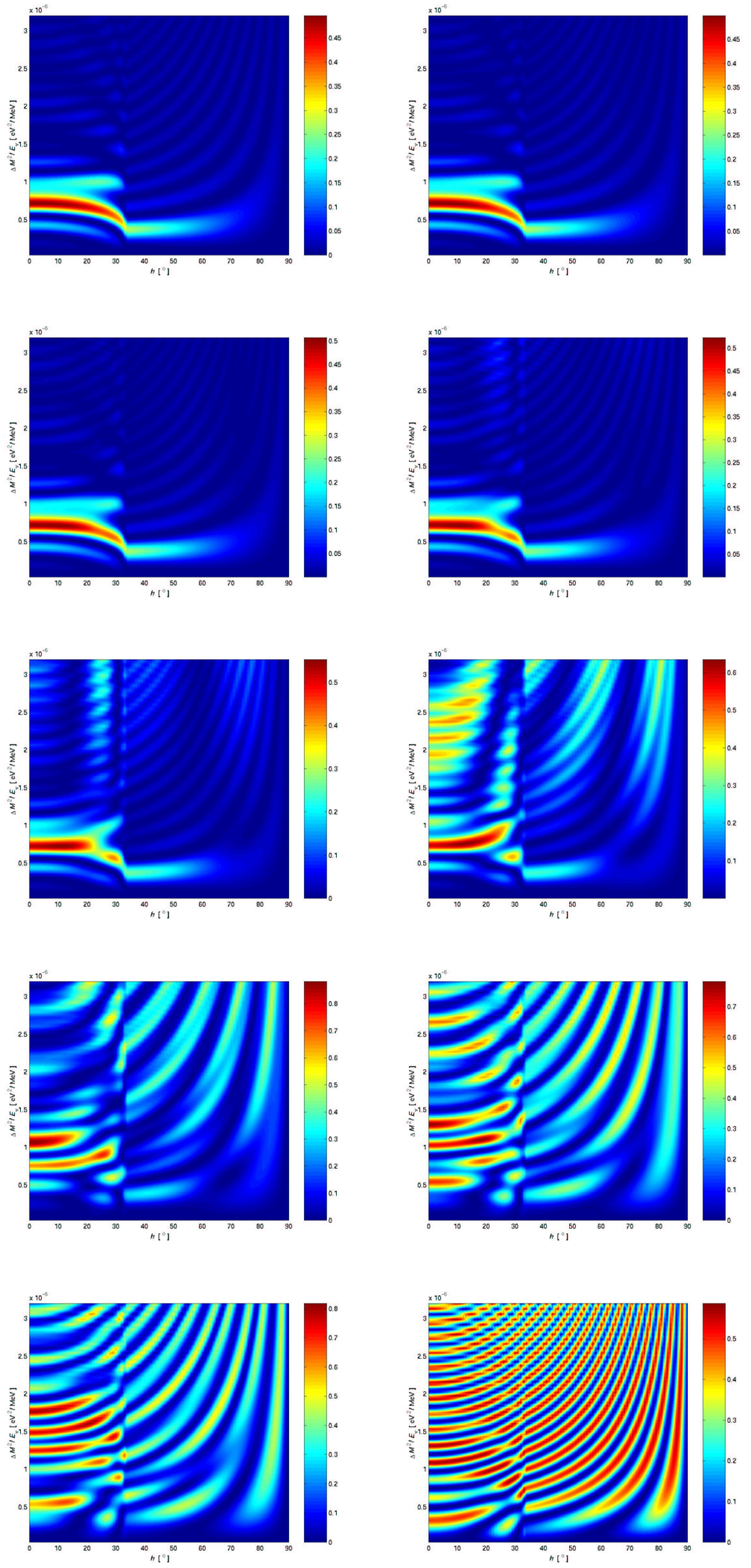

FIG. 5: The (appearance) oscillation transition probability $P_{\mu e}$ as a function of the nadir angle $h$ and $\Delta M^{2} / E_{\nu}$ for varying values of the small mass squared difference $\Delta m^{2}$ [30]. From left to right and from top to bottom, the following ten values of the small mass squared difference have been used: $\Delta m^{2}=0,2 \cdot 10^{-5} \mathrm{eV}^{2}, 5 \cdot 10^{-5} \mathrm{eV}^{2}, 10^{-4} \mathrm{eV}^{2}, 2 \cdot 10^{-4} \mathrm{eV}^{2}, 5 \cdot 10^{-4} \mathrm{eV}^{2}, 10^{-3} \mathrm{eV}^{2}, 1.6$. $10^{-3} \mathrm{eV}^{2}, 2 \cdot 10^{-3} \mathrm{eV}^{2}, 3.2 \cdot 10^{-3} \mathrm{eV}^{2}$. Dark red corresponds to maximal conversion (which is different from figure to figure) and dark blue corresponds to $P_{\mu e}=0$. Parameters: $\theta_{1}=45^{\circ}, \theta_{2}=5^{\circ}, \theta_{3}=45^{\circ}, \delta=0$, and $\Delta M^{2}=3.2 \cdot 10^{-3} \mathrm{eV}^{2}$. 


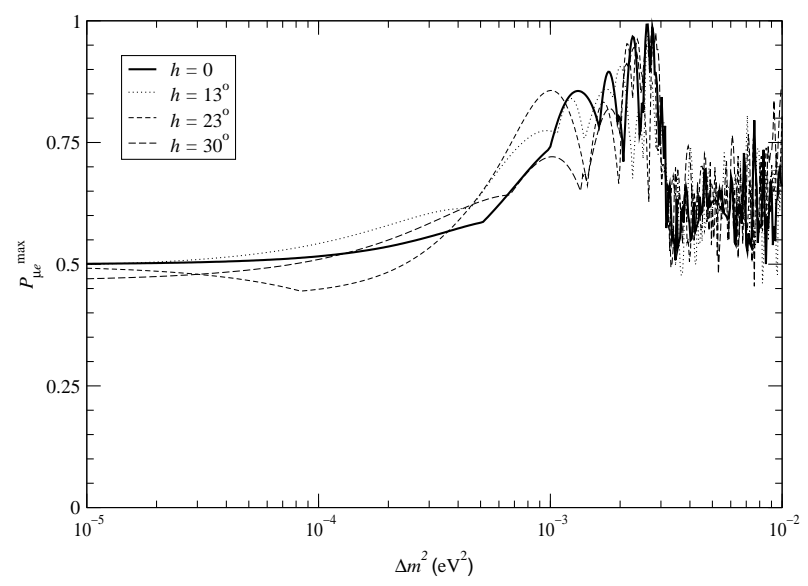

FIG. 6: The maximal (appearance) oscillation transition probability $P_{\mu e}^{\max }$ as a function of the small mass squared difference $\Delta m^{2}$ in the region $0 \leq \theta_{2} \leq 10^{\circ}$ and $1 \mathrm{GeV} \leq E_{\nu} \leq 100 \mathrm{GeV}$ for four different values of the nadir angle $h=0,13^{\circ}, 23^{\circ}, 30^{\circ}$. Parameters: $\theta_{1}=45^{\circ}, \theta_{3}=45^{\circ}, \delta=0$, and $\Delta M^{2}=3.2 \cdot 10^{-3} \mathrm{eV}^{2}$. [30]

Clearly, the oscillatory pattern, the dissolution of the simply connected red area, the drops in the transition probability, and the increase of the maximal transition probability are effects of interference between the mass squared differences $\Delta m^{2}$ and $\Delta M^{2}$. This is solely a three flavor neutrino oscillation model effect, since there is simply no way to obtain such an interference effect in a two flavor neutrino oscillation model, which has only one mass squared difference. Furthermore, the enhancement effects are due to the specific change of matter density from the mantle to the core (and vice versa) and also due to the specific lengths of the neutrino path lengths of the mantle and the core. The mantle-core enhancement effect is a resonance-like effect, but is is not due to a resonance [33].

\section{MATTER OF VARYING DENSITY}

Next, let us shortly describe and discuss the "semi-classical" approximation for neutrino oscillations in matter of varying matter density. So far we have only been looking at:

1. constant matter density profiles or

2. matter density profiles, which can be composed of several layers with constant matter density.

What happens if $A$ is not a constant, i.e., $A=A(r)$, where $0 \leq r \leq L$ ? The answer to this question is that we could use the "semi-classical" approximation method.

The "semi-classical" approximation is obtained by making the following substitution for the evolution operator [34]:

$$
U_{f}(L)=\underbrace{\prod_{i=1}^{n} U_{f}\left(L_{i}, A_{i}\right)}_{n \text { operators }} \rightarrow U_{f}(L)=\underbrace{\bar{\phi} e^{-i L \bar{T}}}_{\text {one operator }},
$$

where $\bar{\phi}$ is a phase factor, $\bar{T} \equiv \overline{\mathscr{H}}_{m}-\left(\operatorname{tr} \overline{\mathscr{H}}_{m}\right) 1_{3} / 3$, and $\overline{\mathscr{H}}_{m} \equiv H_{m}+\bar{A}(L) K_{m}$. Here $\bar{A}(L) \equiv \frac{1}{L} \int_{0}^{L} A(r) d r$ is the average matter density parameter and $K_{m} \equiv \operatorname{diag}(1,0,0)$. Note that the advantage of using this method is that we replace $n$ evolution operators with one single evolution operator evaluated for the average matter density parameter $\bar{A}(L)$ of the considered matter density profile.

The different (reasonable) matter density profiles are the following:

- Constant matter density profiles, i.e., $A(r)=A=$ const. 
- Step function matter density profiles, i.e.,

$$
A(r)=\left\{\begin{array}{l}
A_{1}, 0 \leq r \leq L_{1} \\
A_{2}, L_{1} \leq r \leq L_{1}+L_{2} \\
A_{1}, L_{1}+L_{2} \leq r \leq 2 L_{1}+L_{2}
\end{array},\right.
$$

where $L=2 L_{1}+L_{2}$.

Example: The realistic PREM [46] [35] or Stacey model [25] of the Earth matter density profile can be very well approximated by a mantle-core-mantle step function approximation model [26].

- Linear matter density profiles, i.e., $A(r)=A+B r$. Three flavor neutrino oscillations with linear matter density profiles have been studied by H. Lehmann, P. Osland, and T.T. Wu [36].

- Exponentially decreasing matter density profiles, i.e., $A(r)=A e^{-r / L}$. Three flavor neutrino oscillations with exponentially decreasing matter density profiles have been studied by P. Osland and T.T. Wu [37, 38].

Example: The matter density profile of the Sun can be well-approximated by an exponentially decreasing function of the following form: $\rho_{\odot}(r)=\rho_{\odot}(0) e^{-r / r_{0}}$, where $\rho_{\odot}(0)=200 \mathrm{~g} / \mathrm{cm}^{3}, r_{0}=R_{\odot} / 10.54 \simeq 66000 \mathrm{~km}$, and $R_{\odot} \simeq 6.96 \cdot 10^{8} \mathrm{~m} / \mathrm{s}[39,40]$.

- Power-law matter density profiles, i.e., $A(r)=A r^{-n}$, where $n \in \mathbb{Z}^{+}=\{1,2,3, \ldots\}$.

Example: The matter density profiles for supernovas are proportional to $\frac{1}{r^{3}}$.

It turns out that the "semi-classical" approximation method works well for matter density profiles that are not fluctuating too much.

\section{LONG BASELINE EXPERIMENTS}

In this section, we will briefly mention and summarize some of the LBL experiments that are in operation at present as well as some of the future ones. All these LBL experiments have in common that they are suitable for studying effects of neutrino oscillations in matter.

\section{A. K2K (KEK $\rightarrow$ Super-Kamiokande) $[8,9]:$}

- In operation!

- Channels: $\nu_{\mu} \rightarrow \nu_{\alpha}$, where $\alpha=e, \mu, \tau$.

Almost a pure (99\%) $\nu_{\mu}$ beam from $\pi^{+}$decays, which are generated in the KEK Proton Synchrotron.

- Baseline length and average neutrino energy: $L \simeq 250 \mathrm{~km}$ and $\left\langle E_{\nu}\right\rangle \simeq 1.3 \mathrm{GeV}$.

- Results of data-taking [41]:

- Observed events: 27

- Expected events: $40.3_{-4.6}^{+4.7}$

The K2K data are compatible with the Super-Kamiokande data.

B. MINOS (Fermilab $\rightarrow$ Soudan) $[\mathbf{1 0}-12]:$

- Starts 2003 !

- Channels: $\nu_{\mu} \rightarrow \nu_{\alpha}$, where $\alpha=e, \mu, \tau$.

- Baseline length and average neutrino energies: $L \simeq 730 \mathrm{~km}$ and $\left\langle E_{\nu}\right\rangle=3,6,12 \mathrm{GeV}$.

The baseline length of MINOS is approximately three times longer than that of K2K. One advantage with MINOS (over $\mathrm{K} 2 \mathrm{~K}$ ) is that it can use three different neutrino energies. 


\section{CERN-LNGS (CERN $\rightarrow$ Gran Sasso) [13-15]:}

- Starts 2005 !

- Channels: $\nu_{\mu} \rightarrow \nu_{e}, \nu_{\mu} \rightarrow \nu_{\mu}, \nu_{\mu} \rightarrow \overline{\nu_{\mu}}$, and $\nu_{\mu} \rightarrow \nu_{\tau}$.

- Detectors at Gran Sasso: ICARUS [15] and OPERA [14].

- Baseline length: $L \simeq 732 \mathrm{~km}$.

Although the baseline lengths of MINOS and CERN-LNGS are nearly the same, they have different sensitivities, i.e., $\left\langle E_{\nu}\right\rangle / L\left(\right.$ or $\left.L /\left\langle E_{\nu}\right\rangle\right)$ are different.

\section{SUMMARY AND CONCLUSIONS}

In conclusion, analytical formulas for three flavor neutrino oscillations in matter have been derived using the Cayley-Hamilton formalism. Actually, the Cayley-Hamilton formalism can be used to derive neutrino oscillation formulas in matter for arbitrary numbers of neutrino flavors. These formulas will be exact up to four flavors, since the characteristic equations of degree five or higher are not analytically solvable in general. However, the analytical results are rather lengthy and not so illuminating without making any approximations. The obtained analytical formulas can, however, easily be used in computer programs. One advantage is that no numerical integrations are needed in solving the Schrödinger equation, which should reduce the computational time considerably.

Furthermore, the auxiliary matter mixing parameters have been given in terms of the vacuum mixing parameters and the matter density parameter. It has also been found that interference between the small and large mass squared differences can change the pattern of the neutrino oscillation probabilities drastically. This is a pure three neutrino flavor effect, since if we are using only two neutrino flavors, we only have one mass squared difference. Finally, we found that for some non-constant matter density profiles the "semi-classical" approximation method can be used.

The theoretical developments and the new experimental setups indeed mean an interesting time for neutrino physics and physics in general.

\section{ACKNOWLEDGMENTS}

I would like to thank the local organizing committee and especially Prof. Barbara Badelek for the invitation as a speaker to the 1st Scandinavian NeutrinO Workshop (SNOW) in Uppsala, Sweden, February 8-10, 2001.

Furthermore, I would also like to thank my collaborators Martin Freund, Serguey T. Petcov, and Håkan Snellman for useful discussions and valuable comments.

This work was supported by the Swedish Foundation for International Cooperation in Research and Higher Education (STINT), the Wenner-Gren Foundations, and the "Sonderforschungsbereich 375 für Astro-Teilchenphysik der Deutschen Forschungsgemeinschaft".

\section{REFERENCES}

[1] M. Tegmark and J. A. Wheeler, Scientific American 284(2), 54 (2001), quant-ph/0101077.

[2] Talk given by K. Frame at the 1st Scandinavian NeutrinO Workshop, Uppsala, Sweden, February 8-10, 2001.

[3] BOREXINO Collaboration, F.P. Calaprice, Nucl. Phys. B (Proc. Suppl.) 87, 180 (2000).

[4] MONOLITH Collaboration, A. Geiser, hep-ex/0008067.

[5] Talk given by A. Geiser at the 1st Scandinavian NeutrinO Workshop, Uppsala, Sweden, February 8-10, 2001.

[6] KamLAND Collaboration, A. Suzuki, Nucl. Phys. B (Proc. Suppl.) 77, 171 (1999).

[7] Talk given by Y. Declais at the 1st Scandinavian NeutrinO Workshop, Uppsala, Sweden, February 8-10, 2001.

[8] K2K Collaboration, K. Nishikawa, Nucl. Phys. B (Proc. Suppl.) 77, 198 (1999).

[9] Talk given by Y. Itow at the 1st Scandinavian NeutrinO Workshop, Uppsala, Sweden, February 8-10, 2001.

[10] S. G. Wojcicki, Nucl. Phys. B (Proc. Suppl.) 77, 182 (1999).

[11] Talk given by V. Paolone at the Europhysics Neutrino Oscillation Workshop (NOW 2000), Otranto, Italy, September 9-16, 2000.

[12] Talk given by A. Para at the 1st Scandinavian NeutrinO Workshop, Uppsala, Sweden, February 8-10, 2001.

[13] CERN-LNGS Collaboration, P. Picchi and F. Pietropaolo, Nucl. Phys. B (Proc. Suppl.) 77, 187 (1999). 
[14] Talk given by H. Pessard at the 1st Scandinavian NeutrinO Workshop, Uppsala, Sweden, February 8-10, 2001.

[15] Talk given by A. Rubbia at the 1st Scandinavian NeutrinO Workshop, Uppsala, Sweden, February 8-10, 2001.

[16] Talk given by P.-O. Hulth at the 1st Scandinavian NeutrinO Workshop, Uppsala, Sweden, February 8-10, 2001.

[17] Particle Data Group, D.E. Groom et al., Eur. Phys. J. C 15, 1 (2000), http://pdg.1bl.gov/.

[18] D. Tytler, J. M. O’Meara, N. Suzuki, and D. Lubin, astro-ph/0001318.

[19] Talk given by H. Snellman at the 1st Scandinavian NeutrinO Workshop, Uppsala, Sweden, February 8-10, 2001.

[20] T. Ohlsson and H. Snellman, J. Math. Phys. 41, 2768 (2000), hep-ph/9910546.

[21] T. Ohlsson and H. Snellman, Phys. Lett. B 474, 153 (2000), hep-ph/9912295; 480, 419(E) (2000).

[22] T. Ohlsson, in preparation.

[23] V. Barger, S. Pakvasa, T. J. Weiler, and K. Whisnant, Phys. Lett. B 437, 107 (1998), hep-ph/9806387.

[24] M. Freund, TUM-HEP-404/01, in preparation.

[25] F. D. Stacey, Physics of the Earth (Wiley, 1977), 2nd ed.

[26] M. Freund and T. Ohlsson, Mod. Phys. Lett. A 15, 867 (2000), hep-ph/9909501, and references therein.

[27] S.P. Mikheyev and A.Yu. Smirnov, Yad. Fiz. 42, 1441 (1985), [Sov. J. Nucl. Phys. 42, 913 (1985)].

[28] S.P. Mikheyev and A.Yu. Smirnov, Nuovo Cimento C 9, 17 (1986).

[29] L. Wolfenstein, Phys. Rev. D 17, 2369 (1978).

[30] Y. Farzan, T. Ohlsson, and S. T. Petcov, Ref. SISSA 17/2001/EP, TUM-HEP-406/01, in preparation.

[31] M.C. Gonzalez-Garcia, C. Peña-Garay, and A.Yu. Smirnov, Phys. Rev. D (to be published), hep-ph/0012313.

[32] Talk given by M.C. Gonzalez-Garcia at the 1st Scandinavian NeutrinO Workshop, Uppsala, Sweden, February 8-10, 2001

[33] S. T. Petcov, Phys. Lett. B 434, 321 (1998), hep-ph/9805262; [444, 584(E) (1998)].

[34] T. Ohlsson and H. Snellman, TUM-HEP-405/01, in preparation.

[35] A. M. Dziewonski and D. L. Anderson, Phys. Earth Planet. Inter. 25, 297 (1981).

[36] H. Lehmann, P. Osland, and T. T. Wu, hep-ph/0006213.

[37] P. Osland and T. T. Wu, Phys. Rev. D 62, 013008 (2000), hep-ph/9912540.

[38] Talk given by P. Osland at the 1st Scandinavian NeutrinO Workshop, Uppsala, Sweden, February 8-10, 2001.

[39] J. N. Bahcall, Neutrino Astrophysics (Cambridge University Press, Cambridge, 1989).

[40] J. N. Bahcall, M. H. Pinsonneault, and S. Basu, astro-ph/0010346.

[41] Talk given by J. Hill at the Europhysics Neutrino Oscillation Workshop (NOW 2000), Otranto, Italy, September 9-16, 2000.

[42] Super-Kamiokande Collaboration, T. Toshito, talk given at the XXXth International Conference on High Energy Physics (ICHEP 2000), Osaka, Japan, 2000.

[43] Here a light neutrino is a neutrino with mass less than or equal to $m_{Z^{0}} / 2 \simeq 45 \mathrm{GeV}$, where $m_{Z^{0}}=(91.1882 \pm$ $0.0022) \mathrm{GeV}[17]$ is the mass of the $Z^{0}$ gauge boson.

[44] We use the Super-Kamiokande value of the large mass squared difference $\Delta M^{2}=3.2 \cdot 10^{-3} \mathrm{eV}^{2}[42]\left(\Delta M^{2}=\right.$ $\left.2.5 \cdot 10^{-3} \mathrm{eV}^{2}[9]\right)$.

[45] The best fit point value of the large mixing angle (LMA) solution is $\Delta m^{2}=3.65 \cdot 10^{-5} \mathrm{eV}^{2}\left(10^{-5} \mathrm{eV}^{2} \lesssim \Delta m^{2} \lesssim\right.$ $\left.10^{-3} \mathrm{eV}^{2}\right)[31,32]$.

[46] PREM is the abbreviation for Preliminary Reference Earth Model. 